\title{
Realizing chiral fermions in the type IIB matrix model at finite $N$
}

\author{
Jun Nishimura ${ }^{a b}$ and Asato Tsuchiya ${ }^{c}$ \\ ${ }^{a}$ High Energy Accelerator Research Organization (KEK), \\ Tsukuba, Ibaraki 305-0801, Japan \\ ${ }^{b}$ Department of Particle and Nuclear Physics, \\ Graduate University for Advanced Studies (SOKENDAI), \\ Tsukuba, Ibaraki 305-0801, Japan \\ ${ }^{c}$ Department of Physics, Shizuoka University, \\ 836 Ohya, Suruga-ku, Shizuoka 422-8529, Japan \\ jnishi@post.kek.jp, satsuch@ipc.shizuoka.ac.jp
}

\begin{abstract}
We discuss how chiral fermions can appear in the type IIB matrix model, which is considered to be a nonperturbative formulation of superstring theory. In particular, we are concerned with a constructive definition of the theory, in which we start with a finite$N$ configuration and take the large- $N$ limit later on. We point out that there exists a certain necessary condition which the structure of the extra dimensions should satisfy. As an example, we consider a previous proposal using intersecting branes and show that chiral fermions can indeed be realized in four dimensions by introducing a matrix counterpart of warped space-time. This is remarkable in view of the well-known difficulty in realizing chiral fermions in lattice gauge theory.
\end{abstract}

Keywords: Matrix Models, Superstring Vacua. 


\section{Contents}

1. Introduction 1

2. Lorentz invariant backgrounds 2

3. Mode expansion of fermionic variables 3

4. Explicit example 6

5. Gauge symmetry 9

6. Summary and discussions 9

\section{Introduction}

An important feature of the Standard Model of elementary particles is that fermions are chiral. It is quite nontrivial, however, to get chiral fermions from a fundamental theory in higher dimensions such as superstring theory or from phenomenological models with extra dimensions. Note, for instance, that fermions in higher dimensions become vector-like in four dimensions if one applies simple dimensional reduction. A popular idea among many others is to use orbifolding, which amounts to imposing nontrivial boundary conditions in the extra dimensions.

In this paper we discuss how chiral fermions can appear in the type IIB matrix model [1], which is considered to be a nonperturbative definition of superstring theory. This is highly motivated, in particular, in view of recent developments in the Lorentzian version of the model. In Ref. [2], Monte Carlo studies were made possible, and it was shown that a well-defined large- $N$ limit can be taken despite the fact that the action is not positive definite. Moreover, the time evolution of the space was extracted from the ten bosonic matrices, and it was shown that three out of nine directions start to expand after some critical time, signaling spontaneous breaking of nine-dimensional rotational symmetry. Classical equations of motion are expected to be valid at late times $[3,4]$, and interesting solutions with expanding behavior, which naturally solve the cosmological constant problem, were found [4]. Ref. [5] discussed how one can obtain a local field theory from fluctuations around a classical solution representing a commutative space-time.

The issue of realizing chiral fermions in the type IIB matrix model has been discussed by many authors. For instance, Ref. [6] addressed this issue by considering an "effective theory" for toroidally compactified extra dimensions. The space in extra dimensions is represented by six unitary matrices, and the overlap Dirac operator is used to respect 
chirality. Chiral fermions are indeed realized in the large- $N$ limit of finite- $N$ classical backgrounds with nonvanishing fluxes in extra dimensions. However, the connection between this "effective theory" and the original model is not very clear.

More recently, Ref. [7] proposed to realize chiral fermions in four dimensions using matrix configurations representing intersecting branes. This proposal is based on the original model unlike the one mentioned above. Here we are concerned with a constructive definition, in which we start with a finite- $N$ configuration and take the large- $N$ limit later on. By explicit calculations, we confirm below that a chiral zero mode of the Dirac operator indeed appears on the intersection of the branes. However, we also find that another chiral zero mode with opposite chirality appears. Because of this unwanted zero mode, we obtain a vector-like theory in four dimensions if space-time is a direct product of our four-dimensional space-time and the extra dimensions as the authors implicitly assume.

In fact the above conclusion can be understood as a consequence of a "no-go theorem" that applies to the original model, which is written in terms of Hermitian matrices and with an ordinary Dirac operator. This no-go theorem can be circumvented, however, by considering a matrix counterpart of the warped space-time, which appears generically in the type IIB matrix model. We show that it is indeed possible to realize chiral fermions in four dimensions. This is remarkable in view of the well-known difficulty in realizing chiral fermions in lattice gauge theory. While we need to show eventually that a background that gives rise to chiral fermions appears dynamically, we consider it very promising that such a finite- $N$ configuration does exist in the type IIB matrix model.

The rest of this article is organized as follows. In section 2 we discuss Lorentz invariant backgrounds and show that a matrix version of the warp factor appears in general. In section 3 we expand the fermionic variables in terms of modes in the extra dimension. In particular, we show that one can obtain a chiral fermion in $4 \mathrm{~d}$ if the matrix version of the warp factor satisfies certain conditions. In section 4 we present an explicit example of the background configurations that give rise to a chiral fermion in $4 \mathrm{~d}$. In section 5 we show how one can obtain non-Abelian gauge groups in the present setup, and discuss some issues related to gauge interactions. Section 6 is devoted to a summary and discussions.

\section{Lorentz invariant backgrounds}

In the type IIB matrix model, the space-time is represented by the ten bosonic $N \times N$ Hermitian matrices $A_{M}(M=0, \ldots, 9)$. As it was shown by Ref. [2], an expanding threedimensional space appears dynamically after some time. At later times, it is speculated that three-dimensional space becomes much larger than the Planck scale, and that quantum fluctuations can be neglected at large scales [3,4]. Furthermore, as far as we do not consider too long time scale, we may neglect the expansion of space and therefore the space-time has $\mathrm{SO}(3,1)$ Lorentz symmetry. Thus we are led to consider matrix configurations given by

$$
\begin{gathered}
A_{\mu}=X_{\mu} \otimes M \quad(\mu=0, \ldots, 3), \\
A_{a}=\mathbb{1}_{n} \otimes Y_{a} \quad(a=4, \ldots, 9) .
\end{gathered}
$$


Here we assume that the $n \times n$ Hermitian matrices $X_{\mu}$ have the property $O_{\mu \nu} X_{\nu}=$ $g[O] X_{\mu} g[O]^{\dagger}$, where $O \in \mathrm{SO}(3,1)$ and $g[O] \in \mathrm{SU}(n)$. Then (2.1), (2.2) may be regarded as the most general configuration that is $\mathrm{SO}(3,1)$ invariant up to $\mathrm{SU}(N)$ symmetry.

The Hermitian matrix $M$ in (2.1) may be regarded as a matrix version of the warp factor. ${ }^{1}$ The special case $M=\mathbb{1}$ corresponds to a space-time which is a direct product of $(3+1)$-dimensional space-time and the extra dimensions. However, from the viewpoint of the Lorentz symmetry, there is no reason to set $M=\mathbb{1}$.

\section{Mode expansion of fermionic variables}

The type IIB matrix model has an action with the fermionic part given by

$$
S_{\mathrm{f}}=\frac{1}{2} \operatorname{Tr}\left(\bar{\Psi} \Gamma^{M}\left[A_{M}, \Psi\right]\right),
$$

where $\Gamma_{M}$ are $32 \times 32$ gamma matrices in $10 \mathrm{~d}$. The fermionic matrices $\Psi_{\alpha}(\alpha=1, \ldots, 32)$ are Majorana-Weyl fermions in 10d, and, in particular, they satisfy

$$
\Gamma_{\chi} \Psi=\Psi
$$

where $\Gamma_{\chi}$ is the chirality operator in $10 \mathrm{~d}$.

In order to discuss chiral fermions in $4 \mathrm{~d}$, it is convenient to decompose the gamma matrices in $10 \mathrm{~d}$ into the ones in $4 \mathrm{~d}$ and $6 \mathrm{~d}$ as

$$
\begin{aligned}
& \Gamma^{\mu}=\gamma^{\mu} \otimes \mathbb{1}_{8}, \\
& \Gamma^{a}=i \gamma_{\chi}^{(4 \mathrm{~d})} \otimes \Delta^{a},
\end{aligned}
$$

where $\gamma^{\mu}$ and $\Delta^{a}$ are gamma matrices in $4 \mathrm{~d}$ and $6 \mathrm{~d}$, respectively, which satisfy

$$
\begin{aligned}
\left\{\gamma^{\mu}, \gamma^{\nu}\right\} & =-2 \eta^{\mu \nu}, \\
\left\{\Delta^{a}, \Delta^{b}\right\} & =2 \delta^{a b},
\end{aligned}
$$

and $\gamma_{\chi}^{(4 d)}$ is the chirality operator in $4 \mathrm{~d}$. Note that the chirality operator $\Gamma_{\chi}$ in $10 \mathrm{~d}$ can be decomposed as

$$
\Gamma_{\chi}=\gamma_{\chi}^{(4 \mathrm{~d})} \otimes \Delta_{\chi}^{(6 \mathrm{~d})}
$$

where $\Delta_{\chi}^{(6 \mathrm{~d})}$ is the chirality operator in $6 \mathrm{~d}$.

In the case of quantum field theory in higher dimensions, one decomposes fields into Kaluza-Klein modes, which can then be identified as four-dimensional fields. Here we make a similar analysis in the language of matrices. ${ }^{2}$ We consider expanding the fermionic variables in terms of the eigenmodes of the Dirac operator in $6 \mathrm{~d}$ defined by

$$
D_{6 \mathrm{~d}} \Phi=\Delta^{a}\left[Y_{a}, \Phi\right]
$$

\footnotetext{
${ }^{1}$ This matrix $M$ introduces noncommutativity such as $\left[A_{\mu}, A_{a}\right] \neq 0$ in general. However, as one can see from eq. (3.23), the noncommutativity does not show up in $(3+1)$-dimensional field theory as far as massless modes are concerned.

${ }^{2}$ The analysis presented here is improved from the one given in the first version of this paper, in which we discussed the solutions to the Dirac equation in 10d. In particular, the improved analysis is free from the problem pointed out in the footnote 1 of Ref. [8].
} 
In the explicit example to be discussed in the next section, we consider a configuration of $Y_{a}$, which has a block diagonal form

$$
Y_{a}=\left(\begin{array}{cc}
Y_{a}^{(1)} & 0 \\
0 & Y_{a}^{(2)}
\end{array}\right) .
$$

Correspondingly, we decompose $\Phi$ in eq.(3.6) as

$$
\Phi=\left(\begin{array}{ll}
\Phi^{(1,1)} & \Phi^{(1,2)} \\
\Phi^{(2,1)} & \Phi^{(2,2)}
\end{array}\right) .
$$

Since the Dirac operator $D_{6 \mathrm{~d}}$ acts on each block $\Phi^{(I, J)}(I, J=1,2)$ independently, the eigenvalue problem for $D_{6 \mathrm{~d}}$ can be decomposed into that in each block.

As we will see in the explicit example, chiral fermions actually appear in off-diagonal blocks. Therefore, from now on, we consider the eigenvalue problem for $\varphi \equiv \Phi^{(1,2)}$, which is given by

$$
\Delta^{a}\left(Y_{a}^{(1)} \varphi-\varphi Y_{a}^{(2)}\right)=\lambda \varphi .
$$

Due to the fact that $Y_{a}$ and $\Delta^{a}$ are Hermitian matrices, one can easily show that the eigenvalue $\lambda$ in (3.9) is real. Also, by multiplying $\Delta_{\chi}^{(6 \mathrm{~d})}$ to (3.9) from the left, one obtains

$$
\Delta^{a}\left\{Y_{a}^{(1)}\left(\Delta_{\chi}^{(6 \mathrm{~d})} \varphi\right)-\left(\Delta_{\chi}^{(6 \mathrm{~d})} \varphi\right) Y_{a}^{(2)}\right\}=-\lambda\left(\Delta_{\chi}^{(6 \mathrm{~d})} \varphi\right) .
$$

This implies that if $\varphi$ is an eigenvector with the eigenvalue $\lambda, \Delta_{\chi}^{(6 \mathrm{~d})} \varphi$ is an eigenvector with the eigenvalue $-\lambda$. In particular, $\varphi$ and $\Delta_{\chi}^{(6 \mathrm{~d})} \varphi$ are linearly independent for $\lambda \neq 0$. Therefore we can construct left-handed and right-handed modes by taking linear combinations of $\varphi$ and $\Delta_{\chi}^{(6 \mathrm{~d})} \varphi$ with $\lambda \neq 0$ as

$$
\begin{aligned}
\varphi_{R} & =\frac{1+\Delta_{\chi}^{(6 \mathrm{~d})}}{2} \varphi, \\
\varphi_{L} & =\frac{1-\Delta_{\chi}^{(6 \mathrm{~d})}}{2} \varphi,
\end{aligned}
$$

which satisfy

$$
\begin{aligned}
& \Delta_{\chi}^{(6 \mathrm{~d})} \varphi_{R}=\varphi_{R}, \\
& \Delta_{\chi}^{(6 \mathrm{~d})} \varphi_{L}=-\varphi_{L},
\end{aligned}
$$

and

$$
\begin{aligned}
\Delta^{a}\left(Y_{a}^{(1)} \varphi_{R}-\varphi_{R} Y_{a}^{(2)}\right) & =\lambda \varphi_{L} \\
\Delta^{a}\left(Y_{a}^{(1)} \varphi_{L}-\varphi_{L} Y_{a}^{(2)}\right) & =\lambda \varphi_{R} .
\end{aligned}
$$

Thus the non-zero modes appear in pairs of right-handed and left-handed modes. On the other hand, the zero modes can be assumed to have definite chirality. Since we are 
considering finite- $N$ matrices, the space of $\varphi$ with each chirality has the same dimension. Therefore, the number of zero modes with each chirality should also be the same. However, the actual form of the zero mode $\varphi$ with each chirality can be very different in general. This fact will be important in getting chiral fermions in $4 \mathrm{~d}$.

Let $\left\{\lambda_{n}\right\}$ be a set of non-negative eigenvalues in (3.9). Then we denote the righthanded and left-handed modes corresponding to $\lambda_{n}$ by $\varphi_{n R}$ and $\varphi_{n L}$, respectively. These modes can be normalized in such a way that they satisfy the orthonormal condition

$$
\operatorname{tr}\left(\varphi_{m A}^{\dagger} \varphi_{n B}\right)=\delta_{m n} \delta_{A B}
$$

where $A$ and $B$ are $R$ or $L$.

Now we decompose the fermionic variables $\Psi$ in (3.1) in the same way as in (3.8), and expand the off-diagonal block $\Psi^{(1,2)}$ in terms of the orthonormal basis $\varphi_{n R}$ and $\varphi_{n L}$ constructed above as

$$
\Psi^{(1,2)}=\sum_{n}\left(\psi_{n R} \otimes \varphi_{n R}+\psi_{n L} \otimes \varphi_{n L}\right)
$$

Note that the matrix coefficients $\psi_{n R}$ and $\psi_{n L}$ introduced here satisfy

$$
\begin{aligned}
& \gamma_{\chi}^{(4 \mathrm{~d})} \psi_{n R}=\psi_{n R}, \\
& \gamma_{\chi}^{(4 \mathrm{~d})} \psi_{n L}=-\psi_{n L},
\end{aligned}
$$

as one can see from (3.2), (3.5) and (3.13). Namely, the left-handed and right-handed modes in $6 \mathrm{~d}$ correspond to the left-handed and right-handed modes in $4 \mathrm{~d}$, respectively. Note also that the other off-diagonal block $\Psi^{(2,1)}$ is related to the off-diagonal block $\Psi^{(1,2)}$ that we have considered through charge conjugation as

$$
\left(\Psi^{(2,1)}\right)^{\mathrm{c}}=\Psi^{(1,2)}
$$

due to the Majorana condition for $\Psi$. This allows us to focus only on $\Psi^{(1,2)}$.

In what follows we consider the case in which the matrix warp factor $M$ in (2.1) has a block diagonal form similar to (3.7) as

$$
M=\left(\begin{array}{cc}
M^{(1)} & 0 \\
0 & M^{(2)}
\end{array}\right) .
$$

Then, by substituting (3.16) into the action (3.1), we obtain

$$
\begin{aligned}
S_{\mathrm{f}}= & \sum_{m, n}\left(\operatorname{tr}\left(\bar{\psi}_{m R} \gamma^{\mu} X_{\mu} \psi_{n R}\right) \operatorname{tr}\left(\varphi_{m R}^{\dagger} M^{(1)} \varphi_{n R}\right)-\operatorname{tr}\left(\bar{\psi}_{m R} \gamma^{\mu} \psi_{n R} X_{\mu}\right) \operatorname{tr}\left(\varphi_{m R}^{\dagger} \varphi_{n R} M^{(2)}\right)\right) \\
& +\sum_{m, n}\left(\operatorname{tr}\left(\bar{\psi}_{m L} \gamma^{\mu} X_{\mu} \psi_{n L}\right) \operatorname{tr}\left(\varphi_{m L}^{\dagger} M^{(1)} \varphi_{n L}\right)-\operatorname{tr}\left(\bar{\psi}_{m L} \gamma^{\mu} \psi_{n L} X_{\mu}\right) \operatorname{tr}\left(\varphi_{m L}^{\dagger} \varphi_{n L} M^{(2)}\right)\right) \\
& +i \sum_{n} \lambda_{n} \operatorname{tr}\left(\bar{\psi}_{n R} \psi_{n L}-\bar{\psi}_{n L} \psi_{n R}\right) \\
& +\left(\text { contribution from } \Psi^{(1,1)} \text { and } \Psi^{(2,2)}\right)
\end{aligned}
$$


where we have used (3.3), (3.14), (3.15), (3.17), (3.18) and (3.19).

Let us first consider the case $M=\mathbb{1}$, namely $M^{(1)}=\mathbb{1}$ and $M^{(2)}=\mathbb{1}$ in (3.19). Then the action (3.20) reduces to

$$
\begin{aligned}
S_{\mathrm{f}}= & \sum_{n} \operatorname{tr}\left(\bar{\psi}_{n R} \gamma^{\mu}\left[X_{\mu}, \psi_{n R}\right]+\bar{\psi}_{n L} \gamma^{\mu}\left[X_{\mu}, \psi_{n L}\right]+i \lambda_{n}\left(\bar{\psi}_{n R} \psi_{n L}-\bar{\psi}_{n L} \psi_{n R}\right)\right) \\
& +\left(\text { contribution from } \Psi^{(1,1)} \text { and } \Psi^{(2,2)}\right)
\end{aligned}
$$

which implies that we obtain vector-like fermions in $4 \mathrm{~d}$ in this case. (In section 5, we show that the interaction with the gauge field is also vector-like.) Obviously the same conclusion is reached in the case of $Y_{a}=0$ with general $M$. Therefore we find that in order to obtain chiral fermions, we need to consider $M \neq \mathbb{1}$ and nonvanishing $Y_{a}$. This is a necessary condition for the appearance of chiral fermions in $4 \mathrm{~d}$ in the present setup.

As an example of configurations that give rise to chiral fermions in $4 \mathrm{~d}$, we consider the case with $\lambda_{0}=0$ and $\lambda_{n} \neq 0$ for $n \neq 0$ and with $M^{(1)}$ and $M^{(2)}$ satisfying

$$
M^{(1)} \varphi_{0 L}=\varphi_{0 L} M^{(2)}=\varphi_{0 L}
$$

Then, the action (3.20) reduces to

$$
\begin{aligned}
S_{\mathrm{f}}= & \operatorname{tr}\left(\bar{\psi}_{0 L} \gamma^{\mu}\left[X_{\mu}, \psi_{0 L}\right]\right) \\
& +\sum_{m}\left(\operatorname{tr}\left(\bar{\psi}_{m R} \gamma^{\mu} X_{\mu} \psi_{0 R}\right) \operatorname{tr}\left(\varphi_{m R}^{\dagger} M^{(1)} \varphi_{0 R}\right)-\operatorname{tr}\left(\bar{\psi}_{m R} \gamma^{\mu} \psi_{0 R} X_{\mu}\right) \operatorname{tr}\left(\varphi_{m R}^{\dagger} \varphi_{0 R} M^{(2)}\right)\right)+\text { c.c. } \\
& +\sum_{m, n \neq 0}\left(\operatorname{tr}\left(\bar{\psi}_{m R} \gamma^{\mu} X_{\mu} \psi_{n R}\right) \operatorname{tr}\left(\varphi_{m R}^{\dagger} M^{(1)} \varphi_{n R}\right)-\operatorname{tr}\left(\bar{\psi}_{m R} \gamma^{\mu} \psi_{n R} X_{\mu}\right) \operatorname{tr}\left(\varphi_{m R}^{\dagger} \varphi_{n R} M^{(2)}\right)\right) \\
& +\sum_{m, n \neq 0}\left(\operatorname{tr}\left(\bar{\psi}_{m L} \gamma^{\mu} X_{\mu} \psi_{n L}\right) \operatorname{tr}\left(\varphi_{m L}^{\dagger} M^{(1)} \varphi_{n L}\right)-\operatorname{tr}\left(\bar{\psi}_{m L} \gamma^{\mu} \psi_{n L} X_{\mu}\right) \operatorname{tr}\left(\varphi_{m L}^{\dagger} \varphi_{n L} M^{(2)}\right)\right) \\
& +i \sum_{n \neq 0} \lambda_{n} \operatorname{tr}\left(\bar{\psi}_{n R} \psi_{n L}-\bar{\psi}_{n L} \psi_{n R}\right) \\
& +\left(\text { contribution from } \Psi^{(1,1)} \text { and } \Psi^{(2,2)}\right) .
\end{aligned}
$$

Thus we find that $\psi_{0 L}$ has an appropriate form of the action as a chiral fermion in $4 \mathrm{~d}$, and it does not couple to the other modes. On the other hand, $\psi_{0 R}$ is coupled to the massive

modes with $\lambda_{n} \neq 0$. This implies that we obtain a left-handed chiral fermion in $4 \mathrm{~d}$ but not a right-handed chiral fermion.

\section{Explicit example}

As an example of $Y_{a}$, which allows nontrivial zero eigenvalue in (3.9), we consider a setup representing intersecting branes proposed in Ref. [7]. Here we consider an explicit finite- $N$ configuration given by

$$
Y_{4}=\frac{1}{r}\left(\begin{array}{cc}
L_{1} & 0 \\
0 & \mathbb{1}_{k} \otimes \tilde{L}_{3}
\end{array}\right), \quad Y_{7}=\frac{1}{r}\left(\begin{array}{cc}
0 & 0 \\
0 & L_{2} \otimes \mathbb{1}_{k}
\end{array}\right),
$$




$$
\begin{aligned}
Y_{5} & =\frac{1}{r}\left(\begin{array}{cc}
L_{2} & 0 \\
0 & 0
\end{array}\right), & Y_{8} & =\frac{1}{r}\left(\begin{array}{ll}
0 & 0 \\
0 & \tilde{L}_{3} \otimes L_{1}
\end{array}\right), \\
Y_{6} & =\frac{1}{r}\left(\begin{array}{cc}
\tilde{L}_{3} & 0 \\
0 & L_{1} \otimes \mathbb{1}_{k}
\end{array}\right), & Y_{9} & =\frac{1}{r}\left(\begin{array}{cc}
0 & 0 \\
0 & \mathbb{1}_{k} \otimes L_{2}
\end{array}\right),
\end{aligned}
$$

where the $k \times k$ matrices $L_{i}(i=1,2,3)$ are the $k$-dimensional irreducible representation of $\mathrm{SU}(2)$ algebra, which represents a fuzzy sphere $\sum_{i=1}^{3}\left(L_{i}\right)^{2}=r^{2} \mathbb{1}$ with the radius $r=$ $\frac{1}{2} \sqrt{k^{2}-1}$. In Eq. (4.1) we have also defined $\tilde{L}_{3} \equiv L_{3}+r \mathbb{1}_{k}$ so that the D5-branes represented by the top-left block $\left(Y_{a}^{(11)}\right)$ and the D7-branes represented by the bottom-right block $\left(Y_{a}^{(22)}\right)$ intersect once at the origin of $6 \mathrm{~d}$ space.

We solve Eq. (3.9) for $\varphi$ with $Y_{a}$ given above. Since $\varphi \equiv \Phi^{(1,2)}$ represents the offdiagonal block, it actually corresponds to the degrees of freedom connecting the two different branes. In Fig. 1 the smallest $|\lambda|$ and the second smallest one are plotted against $k$. We find that the smallest one vanishes rapidly with increasing $k$, which implies the appearance of two zero modes with each chirality in the large- $k$ limit. The second smallest $|\lambda|$, on the other hand, seems to approach a non-zero constant, which depends on the parity of $k$.

We define the shape of the wave function for the left-handed and right-handed modes with the smallest $\lambda$ by

$$
\begin{aligned}
& w_{L}(i, j) \equiv \sum_{\alpha}\left|\left(\varphi_{L \alpha}\right)_{i j}\right|^{2}, \\
& w_{R}(i, j) \equiv \sum_{\alpha}\left|\left(\varphi_{R \alpha}\right)_{i j}\right|^{2},
\end{aligned}
$$

respectively. Figs. 2 and 3 shows $w_{L}$ and $w_{R}$, respectively. We see that $w_{L}$ has a peak at $(i, j)=(1,1)$, where $i=1$ and $j=1$ correspond to the intersecting point on the two branes, while $w_{R}$ has a peak at $(i, j)=\left(1, j_{0}\right)$, where $j_{0}=k(k-1)+1$ corresponds to a point on the second brane, which is far from the intersecting point. As the matrix size is increased, the peak of the left-handed mode at $(i, j)=(1,1)$ becomes higher, and all the other components of $w_{L}(i, j)$ vanish in the large- $k$ limit. On the other hand, the peak of the right-handed mode becomes lower as the matrix size is increased.

Due to the simple wave function for the left-handed mode, it is easy to find the matrices $M^{(1)}$ and $M^{(2)}$ which satisfy (3.22) for this mode in the large- $k$ limit. They are given by (e.g., in the case of $k=3$ )

$$
M^{(1)}=\left(\begin{array}{ccc}
1 & 0 & 0 \\
0 & * & * \\
0 & * & *
\end{array}\right)
$$




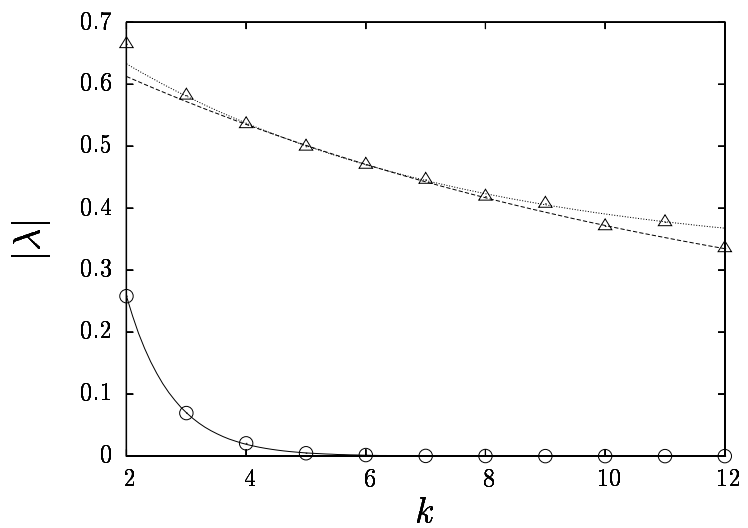

Figure 1: The smallest $|\lambda|$ (circles) and the second smallest one (triangles) are plotted against $k$. The solid, dashed and dotted lines represent the fits to the behavior $|\lambda|=a+b \exp (-c k)$. For the smallest $|\lambda|$, we find $a=0.0001(4)$, which is consistent with zero within the fitting error. For the second smallest one, we find that the fitting curve depends on the parity of $k$, and we obtain $a=0.31(1)$ and $a=0.15(3)$ for odd $k$ and even $k$, respectively.

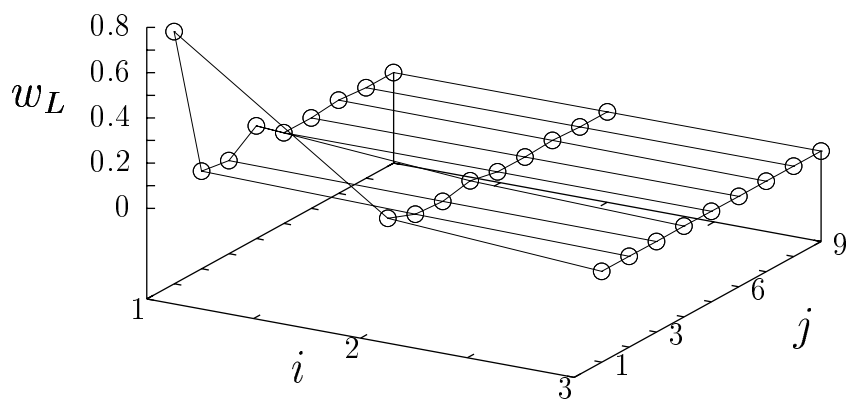

Figure 2: The shape of the wave function for the left-handed mode, $w_{L}(i, j)$. A clear peak is seen at $(i, j)=(1,1)$ already in the $k=3$ case shown here.

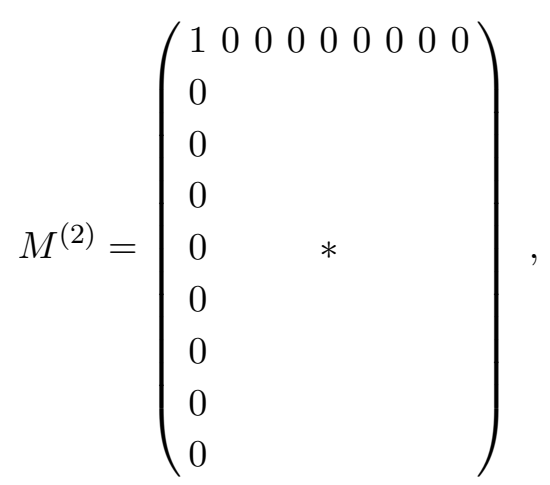

because $\varphi_{L}$ takes the form

$$
\varphi_{L \alpha}=\left(\begin{array}{ccccccccc}
\chi_{\alpha} & 0 & 0 & 0 & 0 & 0 & 0 & 0 & 0 \\
0 & 0 & 0 & 0 & 0 & 0 & 0 & 0 & 0 \\
0 & 0 & 0 & 0 & 0 & 0 & 0 & 0 & 0
\end{array}\right),
$$




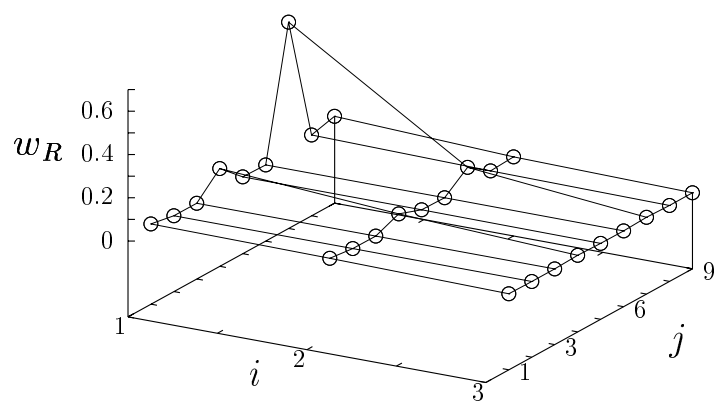

Figure 3: The shape of the wave function for the right-handed mode, $w_{R}(i, j)$, for $k=3$. A peak is seen at $(i, j)=\left(1, j_{0}\right)$, where $j_{0}=k(k-1)+1$.

where $\sum_{\alpha}\left|\chi_{\alpha}\right|^{2}=1$

\section{Gauge symmetry}

We can introduce gauge symmetry by replacing each of the branes by coincident multiple branes similarly to the case of D-brane effective theory. For instance, if we make a replacement

$$
Y_{a}^{(1)} \mapsto Y_{a}^{(1)} \otimes \mathbb{1}_{p}, \quad Y_{a}^{(2)} \mapsto Y_{a}^{(2)} \otimes \mathbb{1}_{q}
$$

in the configuration (4.1), we obtain $\mathrm{U}(p) \times \mathrm{U}(q)$ gauge symmetry as a subgroup of the $\mathrm{U}(N)$ symmetry of the original model. Then the chiral zero mode that appears from the top-right block of $\varphi$ becomes a bifundamental representation $(p, \bar{q})$.

Let us discuss how the chiral zero modes interact with the gauge field. The gauge field is expected to appear from the fluctuation $a_{\mu}$ of $A_{\mu}$ around (2.1). Let us decompose $a_{\mu}$ into blocks as we have done in eq. (3.8). Then the four-dimensional massless modes appear from the diagonal blocks $a_{\mu}^{(I, I)}(I=1,2)$, which can be expanded as $a_{\mu}^{(I, I)}=\sum_{n} \tilde{a}_{\mu n}^{(I)} \otimes b_{n}^{(I)}$ as in eq. (3.16). The gauge field $\tilde{a}_{\mu 0}^{(I)}$ corresponds to the zero mode $b_{0}^{(I)}$, which satisfies $\left[Y_{a}^{(I)},\left[Y_{a}^{(I)}, b_{0}^{(I)}\right]\right]=0$ in the Lorentz gauge. This results in $b_{0}^{(I)} \propto \mathbb{1}$. The gauge field is therefore insensitive to the wave function in the extra dimensions. This implies, in particular, that in the $M=\mathbb{1}$ case the chiral zero modes with opposite chirality that appear from the same block interact with the gauge field in the same manner. Thus we obtain a vector-like gauge theory in that case.

It is not clear how gauge anomaly cancellation can play any role in the present construction. We speculate that a local field theory is obtained at low energy only when the gauge group and the fermion contents are chosen such that the gauge anomaly is canceled. This important issue is left for future investigations.

\section{Summary and discussions}

In this paper we pointed out a necessary condition for the appearance of chiral fermions in the type IIB matrix model. With this new insight, we reconsidered the recent proposal 
based on the idea of intersecting branes. By studying explicit finite- $N$ configurations, we found that unwanted chiral zero modes with opposite chirality appear as anticipated from our general argument. In spite of this problem, we found a simple way to remove the unwanted chiral zero modes. A crucial role was played by a matrix version of the warp factor, which appears generically in the Lorentz invariant background. We emphasize that this is the first explicit example in which chiral fermions in four dimensions are obtained from the type IIB matrix model in the large- $N$ limit starting with finite- $N$ configurations.

An important property of the type IIB matrix model is that it has a built-in mechanism that generates $(3+1)$-dimensional space-time in the Lorentzian case [2]. We consider it very encouraging that the model also allows the appearance of chiral fermions in $(3+1)$ dimensions as the explicit example demonstrates. This gives us strong motivations to proceed to the next step and to think how the background that allows chiral fermions can be generated dynamically. While our discussion given in this paper is not restricted to the Lorentzian case, the signature of the space-time plays an important role in actual dynamics. On top of the determination of the space-time dimensionality, the dominance of a classical configuration at late times is expected in the Lorentzian case $[3,4]$. We therefore consider it important to extend the Monte Carlo studies in Ref. [2] to much later times and/or to study the late time behaviors by classical equations of motion [3,4] with nontrivial structures [9] (and possibly with quantum corrections) in the extra dimensions.

The "warp factor", which we find important in realizing chiral fermions in the type IIB matrix model, may also play important roles in solving the hierarchy problem [10] and in accounting for the difference among three generations of fermions. In this regard it would be interesting to calculate the Yukawa couplings in a manner similar to the work in a closely related field theory [11].

\section{Acknowledgments}

We thank H. Aoki, S.-W. Kim, H. Steinacker and J. Zahn for valuable discussions. Computations were carried out on SR16000 at YITP and FX10 at University of Tokyo. This work is supported in part by Grant-in-Aid for Scientific Research (No. 20540286, 24540264, and 23244057) from JSPS.

\section{References}

[1] N. Ishibashi, H. Kawai, Y. Kitazawa, and A. Tsuchiya, Nucl. Phys. B498, 467 (1997) [hep-th/9612115].

[2] S.-W. Kim, J. Nishimura and A. Tsuchiya, Phys. Rev. Lett. 108, 011601 (2012) [arXiv:1108.1540].

[3] S. -W. Kim, J. Nishimura and A. Tsuchiya, Phys. Rev. D 86, 027901 (2012) [arXiv:1110.4803].

[4] S. -W. Kim, J. Nishimura and A. Tsuchiya, JHEP 1210, 147 (2012) [arXiv:1208.0711].

[5] J. Nishimura and A. Tsuchiya, Prog. Theor. Exp. Phys. 2013, $043 B 03$ (2013) [arXiv:1208.4910]. 
[6] H. Aoki, Prog. Theor. Phys. 125, 521 (2011) [arXiv:1011.1015]; Phys. Rev. D 87, 046002 (2013) [arXiv:1209.4514]; arXiv:1303.3982.

[7] A. Chatzistavrakidis, H. Steinacker and G. Zoupanos, JHEP 1109, 115 (2011) [arXiv:1107.0265].

[8] H. Steinacker and J. Zahn, SIGMA 9, 067 (2013) [arXiv:1309.0650].

[9] H. Steinacker, Prog. Theor. Phys. 126, 613 (2011) [arXiv:1106.6153].

[10] L. Randall and R. Sundrum, Phys. Rev. Lett. 83, 3370 (1999) [hep-ph/9905221].

[11] H. Abe, T. Kobayashi, H. Ohki, A. Oikawa and K. Sumita, Nucl. Phys. B 870, 30 (2013) [arXiv:1211.4317]. 